\title{
La felicidad en entornos de aprendizaje hu- manísticos: el caso del Grado de Historia de la Universidad de Málaga
}

Recibido: 5 de septiembre de 2018 / Revisado: 21 de septiembre de 2018

Aceptado: 27 de noviembre de 2018 / Publicado: 19 de diciembre de 2018

FERNÁNDEZ PARADAS, MERCEDES

Departamento de Historia Moderna y Contemporánea

Universidad de Málaga, España

paradas@uma.es

FERNÁNDEZ PARADAS, ANTONIO RAFAEL

Departamento de didáctica de las Ciencias Sociales

Universidad de Granada, España

antonioparadas@ugr.es

\section{RESUMEN}

Mediante el presente estudio, hemos pretendido analizar el grado de felicidad de los estudiantes del Grado de Historia de la Universidad de Málaga en tres niveles diferentes. Por un lado, su propio grado de felicidad personal, a nivel general. En segundo lugar hemos querido profundizar en el grado de satisfacción que tienen los alumnos al estudiar Historia, y si están contentos con la formación recibida. Finalmente, hemos profundizado en saber si los alumnos que estudian historia, son más felices que aquellos que no la estudian. La investigación realizada, partiendo, de estas tres premisas, pretende responder a la siguiente cuestión: ¿El desarrollo de la conciencia histórica, hace que las personas sean más felices? Para el desarrollo de la investigación se realizó un cuestionario al conjunto de los alumnos matriculados en la asignatura Historia Contemporánea de España (en los turnos de

\section{ABSTRACT}

Happiness in humanistic learning environments: the case of the Degree in History of the University of Málaga

Through the present study, we have tried to analyze the degree of happiness of the students of the Degree of History of the University of Malaga in three different levels. On the one hand, his own degree of personal happiness, on a general level. In the second place we wanted to deepen in the degree of satisfaction that the students have when studying History, and if they are happy with the received formation. Finally, we have deepened in knowing if the students who study history, are happier than those who do not study it. The research carried out, starting from these three premises, aims to answer the following question: Does the development of historical consciousness make people happier? For 
mañana y de tarde), de carácter obligatorio, impartida en el Grado Historia de la Universidad de Málaga durante el curso académico 2017/2018. El cuestionario incluía tres tipos de preguntas: preguntas de respuestas de texto libre; preguntas para elegir en una numérica de satisfacción y preguntas con respuesta de sí o no. Los resultados obtenidos en la investigación nos ha permitido tomar conciencia, no sólo sobre el grado de satisfacción de los alumnos, sino que también nos ha permitido tomar conciencia de sí con el aprendizaje de la historia se puede llegar a ser más feliz. Cabe destacar los resultados obtenidos en relación a los cinco acontecimientos más felices de la historia, ya que los resultados muestran la supervivencia del positivismo en la construcción de la Historia, y la tendencia a construir una historia de carácter beligerante basada en la sucesión de guerras.

Palabras Clave: Felicidad, Historia, educación, enseñanza, enseñanza superior Didáctica, elaboración de medios de enseñanza. the development of the research, a questionnaire was made to the group of students enrolled in the course Contemporary History of Spain (in the morning and afternoon shifts), mandatory, taught in the History Degree of the University of Malaga during the academic year 2017/2018. The questionnaire included three types of questions: free-text answer questions; questions to choose from in a numerical satisfaction and questions with yes or no answer. The results obtained in the research have allowed us to become aware, not only about the degree of satisfaction of the students, but also it has allowed us to become aware of ourselves with the learning of the story we can become happier. It is worth noting the results obtained in relation to the five happiest events in history, since the results show the survival of positivism in the construction of history, and the tendency to build a history of belligerent character based on the succession of wars.

Keywords: Happiness, History, education, teaching, higher education Teaching, development of teaching aids.

\section{Introducción}

Santiago Solanos Gallegos define el concepto felicidad "como el estado de grata satisfacción, espiritual o física. En el ámbito de las organizaciones está la emoción positiva de placer, por lo que se hace o se tiene, viene directamente relacionada con la sensación de alegría como resultado del trabajo realizado. Las personas son felices cuando sienten que su trabajo es la fuente y el origen del bienestar" (Solano Gallegos, 2018, p. 7).

Nuestra intención es aplicar los conceptos The economics of happiness o happiness economics (véanse los trabajos de Frey y Stutzer (1999); Gamero Maldonado (2013)) a nuestro estudio sobre el nivel de felicidad de los estudiantes del Grado de Historia (Vargas y Guerrero, 2017). Como es sabido, the hapiness economic es el campo que analiza a nivel teórico y cuantitativo la felicidad, el bienestar y la satisfacción con la vida, en especial en el trabajo. Sus investigaciones suelen vincular la economía con la psicología, la salud y la sociología, siendo 
menos habitual relacionarlas con la educación. Diversos estudios ponen de manifiesto que las personas con un nivel educativo más alto, suelen alcanzar mayores ingresos económicos y tienen más probabilidad de encontrar mejores empleos (Cuñado y de Gracia, 2012). Sobre los trabajos dedicados a analizar la felicidad de los estudiantes universitarios, véanse, entre otros, a Salgado (2006); Salazar, Larrucha y Flores (2016).

The Happiness Economics ha demostrado que los trabajadores felices son más productivos (Oswald, Proto y Scroi, 2015). El informe de 2016 elaborado por Randstad concluye que los países más productivos, emprendedores e innovadores, Noruega y Estados Unidos, tienen un alto nivel de satisfacción en el trabajo. Esta norma general no se cumple para Japón con un nivel bajo de satisfacción en el trabajo (Randstad, 2016). Aunque con menor intensidad, también es elevado en España, si bien en ese caso parece ser que se explica porque tras la crisis económica se valora mucho el tener empleo (Ravina, Bayardo y Galiano, 2018). La idea de que un empleado feliz es más productivo y creativo se está extendiendo entre las empresas. Prueba de ello es que las empresas están empezando a tomar conciencia de que es positivo que sus trabajadores realicen en su trabajo actividades, como mindfulness, dirigidas a fomentar la concentración que impide focalizar la atención de manera excesiva en los problemas (Khoury, 2013). En 2014 Google fue una de las primeras multinacionales en incorporar cursos de mindfulness para promover una atmósfera positiva y mejorar la inteligencia emocional de sus empleados (Mirabai, 2014).

Pretendemos indagar sobre el nivel de felicidad de los estudiantes universitarios (Santamaría, 2018), en concreto del Grado de Historia de la Universidad de Málaga. Es un colectivo singular, ya que su posterior trabajo como docentes y/o investigadores en ese ámbito de las humanidades puede tener efectos positivos sobre el nivel de conciencia histórica de la sociedad, así cómo la capacidad de esta para tomar decisiones fundamentadas en enseñanzas basadas en situaciones parecidas del pasado. Para conseguir nuestros objetivos, pretendemos responder al siguiente interrogante: ¿cabe establecer una relación directa entre el trabajo que desarrollan los estudiantes y su nivel felicidad? (La felicidad hace posible la creatividad y la productividad, no a la inversa) (Ravina, Villena y Gutiérrez Montoya, 2017). En definitiva, queremos realizar una primera aproximación entre el nivel de felicidad y satisfacción en el trabajo de los alumnos de la titulación de Historia. Por ello, consideramos fundamental examinar su grado de motivación para cursar dichos estudios, el ambiente de trabajo, su compromiso, sus expectativas para encontrar empleo relacionado con sus estudios y su percepción acerca de cómo puede contribuir el conocimiento histórico a que sean más felices (Hernández, Díaz Negrín y Díaz Bolaños, 2018), (Betti, 2018), (Pulido y Herrera, 2018). 


\section{Material y método}

Para el desarrollo de la investigación se realizó un cuestionario al conjunto de los alumnos matriculados en la asignatura de Historia Contemporánea de España (en los turnos de mañana y de tarde), de carácter obligatorio, impartida en el Grado Historia de la Universidad de Málaga durante el curso académico 2017/2018. El cuestionario incluía tres tipos de preguntas: preguntas de respuestas de texto libre; preguntas para elegir en una escala numérica de satisfacción y preguntas con respuesta de sí o no. En función de estas preguntas, se ha pretendido realizar un cuestionario de carácter cuantitativo y cualitativo, que permite contextualizar los datos numéricos en un contexto más profundo. Las preguntas se agrupan en tres bloques. Por un lado, aquellas relativas cuestiones generales de categorización y sus grado de satisfacción personal; en segundo lugar, aquellas que tienen que ver con su grado de satisfacción personal académica, en función de los estudios recibidos; y finalmente aquellas que tiene que ver con el desarrollo de la conciencia histórica y la felicidad (Tello, 2017).

La encuesta consta de 29 preguntas, en las que hemos pretendido reunir información sobre los siguientes aspectos:

1. Perfil-es de los encuestados.

2. Definición del concepto felicidad.

3. ¿Por qué has elegido cursar el Grado de Historia?

4. Nivel de felicidad personal.

5. Nivel de felicidad como alumno-a del Grado de Historia.

6. Si considera que su nivel de felicidad personal afecta a su rendimiento como estudiante del Grado de Historia.

7. ¿Estás satisfecho con el trabajo que haces como estudiante del Grado de Historia?

8. ¿Estas motivado para avanzar en tu aprendizaje en el Grado de Historia?

9. En general, ¿el profesorado favorece o no el aprendizaje del alumno?

10. Si, durante la titulación, se le está formando adecuadamente para incorporarse al mercado laboral desarrollando una profesión relacionada con el Grado de Historia.

11. Nivel de compromiso como estudiante del Grado de Historia.

12. El ambiente de trabajo en el aula favorece las buenas relaciones con otros estudiantes.

13. Si, en general, el profesorado del Grado de Historia es justo al evaluar el trabajo del alumnado. 
14. Si trabajar en equipo le ayuda a mejorar sus conocimientos y/o competencias del Grado de Historia.

15. Si tiene dificultades para trabajar en equipo.

16. Si piensa que sabe trabajar en equipo con sus compañeros.

17. Si considera que estar cursando el Grado de Historia, al permitirle tener conciencia histórica del pasado, le está ayudando a ser más feliz.

18. ¿Una sociedad con mayor conocimiento histórico será una sociedad más feliz?

19. Enumera los cinco acontecimientos más felices de la historia.

20. Si existe relación entre nivel de lectura y felicidad.

21. Si existe relación entre ser más feliz y ser creyente, agnóstico o ateo.

22. Si es voluntario en alguna $\mathrm{ONG}$ o institución.

23. Si debería aprender técnicas de relajación, control y gestión de las emociones.

La encuesta, cumplimentada por alumnos de $3^{\circ}$ del Grado de Historia de la Universidad de Málaga, es representativa ya que han respondido el $34,5 \%$ de los matriculados en ese curso y el 12,5\% en la titulación. El cuestionario fue realizado durante los meses de febrero y marzo de 2018.

\section{Resultados}

La mayoría de los encuestados, el 61,3\%, son hombres, y el 38,7\% mujeres. Casi el 67\% tiene entre 20 y 22 años, el $25,7 \%$ de 23 a 25 años, el 8,1\% de 26 a 30 años y el 2,7\% de 31 a 40 años. Por tanto, en su inmensa mayoría son jóvenes los que han respondido al cuestionario. Casi el 99\% ha accedió a los estudios universitarios desde el Bachillerato.

La Tabla 1 recoge cómo han definido el concepto felicidad. Hemos suprimido aquellas definiciones que eran parecidas. Como se observa, se trata de un concepto subjetivo, la mayoría suele considerarlo como un estado de ánimo que casi la mitad de ellos relacionan con estar bien con independencia de las circunstancias y con la salud.

\begin{tabular}{|c|}
\hline DEFINICIONES \\
\hline Estado de ánimo por el que te sientes increíble. \\
\hline La felicidad es un estado de ánimo que permite a una persona estar en plenitud consigo misma y con su alre- \\
dedor.
\end{tabular}


Estar contento.

Estar bien sin que nos afecten cosas innecesarias.

La felicidad es algo relativo, y en el mayor de los casos es un concepto que viene dado por la realidad material, esto es, la clase social a la que perteneces. La felicidad es estar bien con uno mismo, disfrutar de las cosas olvidando todo lo demás, pues demuestra que se está contento con lo que se está haciendo, generando, además, un bienestar en todo el cuerpo.

Estado de bienestar y tranquilidad de una persona, el cual puede deberse a factores externos o a la propia actitud y mentalidad de la persona.

\section{Salud.}

Consiste en tener confianza y gusto de uno mismo y las personas que te importan.

Ausencia de angustia por el desarrollo personal.

Vivir la vida.

Estado de ánimo, donde no importa lo malo, siempre se ve con una perspectiva positiva.

La felicidad es disfrutar de cada momento, por muy pequeño y rutinario que parezca, aprovechando los buenos momentos y aprendiendo de los malos.

Aprobar todas las asignaturas y no tener que ir a septiembre.

Constructo social que se basa en definir el grado de bienestar personal y placer que una persona tiene en un determinado momento basado en unas necesidades preestablecidas por la presión social.

Tranquilidad mental con uno mismo y con las personas que aprecias.

La felicidad es el sentimiento que sientes cuando vives como quieres, cuando tienes la fortuna de que los tuyos tengan salud y te sientes realizado.

La felicidad es la capacidad de adaptarse a las circunstancias.

Tabla 1. Definiciones de Felicidad por los estudiantes del Grado de Historia de la Universidad de Málaga.

Fuente: Cuestionario sobre la Felicidad y el Grado de Satisfacción de los estudiantes del Grado de Historia de la Universidad de Málaga.

Les hemos preguntado al alumnado acerca de su nivel de felicidad personal en una escala del 1 al 10, siendo este último el valor máximo (Tabla 2). La inmensa mayoría, casi el 78\%, considera que su nivel de felicidad es alto ya que lo sitúa entre 7 y 10 . Empero, es llamativo el porcentaje de los que no alcanzan el valor 5, el 14,4\%. Aún más interesante resulta comparar estos datos con los de su felicidad como Estudiantes ya que constatamos un empeoramiento de los mismos. Si bien el porcentaje de los que no superan el 4 es ligeramente inferior $(13,1 \%)$, el 27,6\% han optado por situarse entre 5-6, es decir, un 
tanto por ciento muy superior respecto de los que optan por estos mismos dígitos al valorar su felicidad personal (7,8\%). Además, los que estarían más satisfechos, del 7 al 10, solo suman el 59,3\% frente al $77,8 \%$ si nos referimos a la felicidad personal. En definitiva, en general los alumnos y alumnas consideran que son más felices a nivel personal que como estudiantes.

\begin{tabular}{|c|c|c|c|}
\hline Niveles & Nivel de felicidad personal & $\begin{array}{c}\text { Nivel de felicidad } \\
\text { como estudiante }\end{array}$ & $\begin{array}{c}\text { Nivel de satisfacción con el trabajo } \\
\text { desarrollado como estudiante }\end{array}$ \\
\hline 1 & 2,2 & 2,6 & 6,6 \\
\hline 2 & 3,0 & 1,3 & 2,6 \\
\hline 3 & 3,9 & 5,3 & 6,6 \\
\hline 4 & 5,3 & 3,9 & 7,9 \\
\hline 5 & 3,9 & 10,5 & 14,5 \\
\hline 6 & 3,9 & 17,1 & 10,5 \\
\hline 7 & 27,9 & 23,7 & 18,4 \\
\hline 8 & 28,9 & 19,8 & 17,2 \\
\hline 9 & 17,1 & 11,9 & 11,8 \\
\hline 10 & 3,9 & 3,9 & 3,9 \\
\hline Total & $\mathbf{1 0 0}$ & $\mathbf{1 0 0}$ & $\mathbf{1 0 0}$ \\
\hline
\end{tabular}

Tabla 2. Nivel de Felicidad personal y como estudiante, y grado de satisfacción con el trabajo como alumno del Grado de Historia de la Universidad de Málaga, en \%. Fuente: Cuestionario sobre la Felicidad y el Grado de Satisfacción de los estudiantes del Grado de Historia de la Universidad de Málaga. Elaboración propia.

¿Cuáles pueden ser los motivos? Para responder a esta cuestión, les hemos inquirido por qué han elegido cursar el Grado de Historia. Pues bien, el 65,8\% dice que por vocación y el 14,5\% porque le gusta la docencia. Esto es, el 80,3\% de los entrevistados habría accedido a la carrera con un elevado grado de motivación. De los restantes, el 5,3\% confiesa que, al no haber sido admitido en el grado que quería cursar, optó por matricularse en Historia, el 3,9\% porque la nota de acceso a este Grado es baja (5), el 1,3\% porque la titulación de Historia permite acceder a determinados puestos de la administración pública y el $9,2 \%$ por otras razones. Es decir, el 19,7\% no parece que esté motivado en su aprendizaje como estudiante del Grado de Historia. Los alumnos, al preguntarles si piensan que su nivel de felicidad personal afecta a su rendimiento como estudiante, el $88,2 \%$ responde afirmativamente y el 11,8\% negativamente.

En cuanto al nivel de satisfacción del alumnado con el trabajo que desarrollan como estudiantes del Grado (Tabla 2), su percepción es mucho más negativa que la que expresan sobre sus niveles de felicidad personal y como estudiante. El 23,7\% se autoevalúa por debajo del 5, el $25 \%$ entre 5 y 6 y el 
$51,3 \%$ entre 7 y 10 . Por tanto, casi la mitad manifiesta que no trabaja tanto como debiera, lo que refleja una escasa implicación, máxime si tenemos en cuenta que más del $80 \%$ afirma que ha elegido la carrera por vocación y porque le gusta la docencia.

Para profundizar en el grado de motivación del alumnado, les hemos preguntado si están motivados para avanzar en su aprendizaje. La respuesta dada mayoritariamente, el 86,6\% dice que sí, no es congruente con su contestación al inquirirles sobre su satisfacción con el trabajo que realizan. Lo que podría indicar que en realidad no están tan motivados o que se conforman con obtener unos resultados de aprendizaje no excesivamente positivos.

Para tener más elementos de juicio, hemos indagado acerca del entorno en que desarrollan su aprendizaje. Para ello, les hemos interpelado si en general el profesorado lo favorece. El 57,9\% afirma que sí, el 42,1\% que no. A nuestro modo de ver, este último porcentaje es demasiado elevado, lo que podría indicar, o bien que los docentes, como colectivo, no estarían realizando lo suficientemente bien su trabajo o que el estudiante no es receptivo a aprender. Hay que señalar que su respuesta tampoco es muy satisfactoria al preguntarles si el docente es justo al evaluar su trabajo, ya que el 55,3\% responde que sí y el 44,7\% que no. En relación a ello, al preguntarles si el ambiente de trabajo en el aula promueve las buenas relaciones con los compañeros, el 68,4\% contesta positivamente, el 31,6\% que no. De nuevo, esta última cifra es preocupante porque indicaría que casi para un tercio el contexto en el que desarrolla buena parte de su actividad como discente no es favorable. En relación a ello, también puede contribuir su opinión acerca de si se les está formando adecuadamente para incorporarse al mercado laboral, con una profesión vinculada con el Grado de Historia. Casi el $82 \%$ dice que no, lo que sin duda contribuirá a que piensen que no están aprovechando plenamente sus estudios.

Uno de los factores que redunda en la creatividad y, por ende, en la felicidad es el trabajo en equipo. Por este motivo, les hemos planteados varias cuestiones sobre este asunto. Al inquirirles si consideran que trabajar en equipo les ayuda a mejorar sus conocimientos y/o competencias del Grado de Historia, el 59,2\% responde que sí y el 40,8\% que no. Es decir, un porcentaje elevado no lo valora como una forma de trabajar positiva, por lo que intentarán en la medida de lo posible evitarla, lo que no redundará en que sean más creativos. Máxime cuando casi el $74 \%$ piensa que no tiene dificultades para trabajar en equipo y el $81,2 \%$ que sabe trabajar en equipo. Al respecto, es posible que buena parte del alumnado estime que el trabajo en equipo, al depender de los demás, puede contribuir a que baje su calificación o bien que otros compañeros del grupo no estén dispuestos a trabajar como debieran, una queja que suele ser habitual al plantearles que realicen trabajos con otros estudiantes.

Otro aspecto a considerar es cómo puede influir el nivel de conciencia histórica en ser feliz. Les hemos preguntando si piensan que el estar cursando el Grado de Historia les permite tener una conciencia 
histórica del pasado, y, como consecuencia de ello, les está ayudando a ser más felices. El 71,1\% dice que sí, el 28,9\% que no. Esto significa que para ellos el cursar el Grado de Historia es beneficioso. Cuando les planteamos la misma pregunta para el conjunto de la sociedad, baja el porcentaje de respuestas positivas al $68,4 \%$.

Además, les hemos pedido que nos digan los 5 acontecimientos más felices de la historia. Un ejemplo de los acontecimientos históricos más felices propuestos sería los siguientes (extracto):

Enumera los cinco acontecimientos más felices de la Historia:

1. La caída del muro de Berlín, la mujer obtiene el derecho a votar, abolición de la esclavitud, fin Segunda Guerra Mundial, el descubrimiento de las vacunas.

2. Elección del Rey, nacimiento de los reyes.

3. El nacimiento de los derechos naturales entre los siglos XI y XII; el abrazo de Vergara de 1839; la tregua de navidad de 1914; la consolidación de la segunda república en España en 1931 o la caída del muro de Berlín de 1989.

4. Fin de la guerra civil española. Voto femenino. Fin de las dos guerras mundiales. Fin de la esclavitud. La creación de la electricidad.

5. La revolución soviética, la revolución cubana, la revolución francesa (1789-1799), la fase inicial del estallido de la guerra civil española (1936-1939), sobre todo en el bando republicano, plasmado en el entusiasmo de los milicianos voluntarios, a los que se puede ver con sus familias o sus compañeros del frente en las fotografías. También la guerra de independencia (1808-1814), plasmado en las tácticas de guerrillas surgidas directamente de la espontaneidad popular y el sentimiento de defensa. Y en definitiva, todo momento histórico en que la propia población fue dueña de su destino sin ninguna intervención de intermediarios.

6. Mayo del 68, la caída del muro de Berlín, el final de las Guerras Mundiales, el discurso de Martin Luther King, la independencia de las colonias durante el siglo XX.

7. La revolución neolítica, la destrucción de Cartago, el descubrimiento de América, la revolución rusa y la invasión de Checoslovaquia de 1968.

8. La abolición de la esclavitud, la muerte de Franco, la llegada de la democracia, el derecho al voto femenino, la legalización del divorcio y los matrimonios homosexuales...

9. El desarrollo de la política romana.2. Los diferentes códigos de igualación de todos los individuos componentes de una sociedad en la Edad Media. 3. Las revoluciones liberales.4. Las revueltas obreras. 5. En la historia actual, el esfuerzo llevado a cabo tan solo por el pueblo que 
intenta conseguir una sociedad más justa (sufragio universal, movimientos subversivos (contra figuras que se aprovechan de su posición social nata o bien de la política para robar) a pesar de la censura y las consecuencias penales...).

10. El fin de la segunda guerra mundial, la caída del muro de Berlín, el sufragio femenino, la abolición de la esclavitud, el fin de la dictadura en España.

11. Todo acontecimiento que tenga que ver con la liberación de cualquier persona de la opresión de la metrópoli, del capitalismo y del patriarcado. De la misma manera la culminación de un proyecto a largo plazo también es motivo de felicidad. Así las cosas, no puedo enumerar 5 sino 5.000 .

12. El fin de la II Guerra Mundial; la caía del muro de Berlín; el descubrimiento de tratamientos médicos; la abolición de la esclavitud; el derecho a voto para la mujer.

13. 1-Final de la Primera Guerra Mundial, 2-La caída del muro de Berlín, 3-Abolición de la esclavitud, 4-Final de la Segunda Guerra Mundial, y 5-la creación de Internet.

14. 1-Obtención de derechos políticos por parte de la mujer. 2. Lucha racial en EEUU. 3. Nacimiento de la escritura. 4. Publicación en 1843 de "Unión Obrera" de Flora Tristán. 5. Establecimiento de la democracia española.

15. Final Segunda Guerra Mundial, descubrimiento penicilina, llegada del hombre a la Luna, invención del cinematógrafo y victoria de España en el mundial de fútbol de 2010.

16. Invención del fuego, de la electricidad, Descubrimiento de la penicilina, Revolución Rusa (1917) y revolución cubana (1959).

17. Todos los avances médicos y tecnológicos.

18. No creo estar capacitada para establecer los acontecimientos más felices de la Historia, además, los acontecimientos no siempre provocan felicidad a todos, pero por como afectaron a la vida de muchas personas, creo que podrían ser, entre otros, los siguientes: La caída del muro de Berlín, la marcha sobre Washington de Martin Luther King, el movimiento "me too" contra el acoso sexual y la consecución del sufragio universal en todos los países donde se ha conseguido, cada uno en su momento de la historia. Ahora mismo solo se me ocurren esos 4.

19. Descubrimiento de fármacos, descubrimientos tecnológicos cotidianos y no tanto en general.

$\mathrm{Al}$ respecto, cabe reseñar que más del 80\% de los hechos seleccionados (77 alumnos respondieron a encuesta en total) se centran en los siguientes temas: el fin de guerras; los avances en los derechos humanos, en especial el voto de la mujer y el fin de la esclavitud; la caída del muro de Berlín en 1989; la recuperación de la democracia en nuestro país; y los avances tecnológicos y médicos. Los resultados de esta 
pregunta, son terriblemente interesantes, ya que en las respuestas ofrecidas subyace toda una perpetuación ideológica que viene produciéndose en el currículo relativo a la historia desde la educación primaria obligatoria, donde los contenidos históricos quedan incluidos en la asignatura didáctica de las Ciencias Sociales (Fernández, Ravina y Gutiérrez, 2018). Es una constancia, desde los niveles básicos de la educación obligatoria española, hasta la propia universidad, inculcar a los alumnos una conciencia histórica que entiende la historia como un proceso beligerante donde la historia se mide, y enseña, en función de las grandes guerras de la historia, que son enseñadas de una manera netamente positivista, con un comienzo y un fin. Guerras producidas y contadas por hombres. Se trata de una visión netamente política de la historia. Los manuales de texto de cualquiera de las etapas educativas españolas, reproducen hasta la saciedad estos patrones de comportamiento, por lo que los resultados de la encuesta, reflejan a la perfección años de currículo histórico basados en guerras. La inmensa mayoría de las respuestas recogidas en este apartado tienen que ver con guerras, la I y II Guerra Mundial, la Guerra Fría, la Guerra Civil Española, la Guerra de Cuba, la Guerra de Independencia, etc. Está claro, que el que se termine una guerra, es un motivo de felicidad y euforia colectiva, pero también es cierto, que probablemente la omisión de otros hechos felices no beligerantes, tenga que ver con el propio desconocimiento de los mismos. Sólo en algunas casos, se han mencionado los descubrimientos médicos, la consecución de los derechos y libertados para las mujeres y los homosexuales, o el descubrimiento del fuego, cuya invención se menciona varias ocasiones.

También les hemos inquirido sobre su nivel de lectura, ya que diversos estudios concluyen que los lectores suelen ser más felices y más optimistas (Ordine y Flexner, 2013). El 85,8\% de los estudiantes afirma que le gusta leer. En cuanto al nivel de frecuencia de libros que leen no relacionados con el Grado de Historia, el 17,1\% dice que al menos lee 1 libro al mes, el 44,7\% entre 1 y 5 libros al año, el 30,3\% al menos 6 libros al año y el 7,9\% no lee. Además, también más del 85\% considera que leer libros no relacionados con sus estudios les ayuda a ser feliz. Su interés por la lectura es mayor que la pauta general del país, ya que más de un tercio de los españoles confiesa que no leen (González, 2016). Otro aspecto que suele vincularse con la felicidad es el ser creyente, ya que diversas investigaciones concluyen que este colectivo suele ser más feliz que los ateos (Reino Unido, 2012), (las personas religiosas, 2014).

El alumnado del Grado de Historia, en concreto más del 80\%, considera que el ser creyente, ateo o agnóstico no influye en ser más o menos felices, lo cual es indicativo del elevado grado de secularización de nuestra sociedad (Ortiz Carmona, 2017), según el cuestionario realizado el 61,8\% de ellos manifiesta que no son creyentes.

Les hemos preguntado acerca de su compromiso con la sociedad, en concreto si son voluntarios en organizaciones no gubernamentales e instituciones puesto que la dedicación a los demás también contribuye a la felicidad, el $21,1 \%$ lo es, el 78,9\% no. Un tanto por ciento muy superior al promedio del país ya que se estima que el 7,9\% de la población mayor de 18 años es voluntaria (Hechos y cifras, 2016) 
Por último, al inquirirles si deberían aprender técnicas de relajación, meditación, control y gestión de las emociones, casi el 74\% se manifiesta a favor, lo que indicaría que son proclives a mejorar sus niveles de bienestar.

\section{Discusión y conclusiones}

La inmensa mayoría de los estudiantes del Grado de Historia de la Universidad de Málaga, el $77,8 \%$, considera que su nivel de felicidad es alto, entre 7 y 10 . Su opinión acerca de su nivel de felicidad como alumno es más negativa, ya que solo el 59,3\% lo sitúan entre 7 y 10 . No parece que este peor dato se deba a falta de motivación, al menos al acceder a la titulación, ya que más del 80\% de los encuestados la han elegido por vocación o porque quieren ser docentes (Montenegro, Córdoba y García, 2017).

En lo concerniente al nivel de satisfacción del alumno con el trabajo que realiza como estudiante del Grado, casi el 24\% se autoevalúan por debajo del 5, y el 25\% entre 5 y 6 . Es decir, muestran poco interés en formarse, lo que se contradice con la motivación que dicen tener cuando comienzan sus estudios.

Respecto al entorno en el que desarrollan su aprendizaje, no parece que sea especialmente favorable. Por ejemplo, solo el 57,9\% considera que el profesorado favorece su aprendizaje y más del $31 \%$ que el ambiente en clase no fomenta las buenas relaciones con otros alumnos. El trabajo en equipo favorece la creatividad. Sin embargo, casi el $41 \%$ de los estudiantes no piensa que les ayuda a mejorar sus conocimientos y/o competencias.

Sí es positiva su percepción acerca de que el conocimiento histórico contribuye a que sean más felices. Asimismo, el hecho de que, en general, les guste leer y practiquen dicho hábito les debe ayudar a ser más felices. Otro aspecto interesante a destacar es que la mayoría dice ser ateo, sin que consideren que ello les hace menos felices. Su nivel de implicación con la sociedad parece ser mayor que el del promedio del país, ya que más del $21 \%$ son voluntarios. Por último, muestran una actitud positiva a aprender técnicas que les permitan relajarse y gestionar sus emociones (Guijón, 2017).

\section{Referencias}

Ahumada Tello, E. (2017). Percepción de competitividad personal. Un estudio desde la perspectiva de la felicidad, el bienestar y la educación en egresados universitarios. Revista de Estudios Empresariales, 1, pp. 34.54.

Betti, C. (2018). La felice esperieza scolastica de un prete, "obbedientissimo ribelle". Historia y Memoria de la Educación, 7, pp. 235-268. 
Bisquerra Alzin, R. y Hernández Paniello, S. (2017). Psicología positiva, educación emocional y el Programa de Aulas Felices. Papeles del Psicólogo, 38 (1), pp. 58-65.

Bush, M. (2014). What's it Like to Take Google's Mindfulness Training. Mindfulness, healthy mind, halthy life. Recuperado de: https://www.mindful.org/whats-it-like-to-take-googles-mindfulness-training/

Cuñado, J. y De Gracia, F. (2012). Does education affect happiness? Evidence for Spain”. Social Indicators Research: An International and Interdisciplinary Journal for Quality-of-Life Measurement, 1, 185-196.

Fernández Paradas, A. R., Ravina Ripoll, R. y Gutiérrez Montoya, G. A. (Coords). (2018). La enseñanza de las Ciencias Sociales en la Educación Primaria Española. Un análisis Holístico de los libros de texto desde el Franquismo hasta la actualidad. El Salvador: Universidad Salesiana

Frey, B.S. y Stutzer, A. (1999). Measuring Preferences by Subjective Well-Being. Journal of Institutional and Theorical Economnics, 155 (4), 755-778.

Gamero Maldonado, H.J. (2013). La Satisfacción Laboral como Dimensión de la felicidad. Ciencia \& Trabajo, 15 (47), 94-102.

González, L. (2017). Hábitos lectores y políticas habituales de lectura. En: MILLÁN, J.A. (coord.). La lectura en España. Informe 2017 (81-91). Madrid: Federación de Gremios de Editores de España.

Guijón Espinosa, V. (2017). La percepción del profesorado sobre felicidad. Tesis doctoral. Granada: Universidad de Granada.

Hechos y cifras 2015. (2016). Recuperado de http://www.prodes.org/Hechos\%20y\%20cifras\%20 del\%20voluntariado.pdf

Hernández Etala, D., Díaz Negrín, M. E. y Díaz Bolaños, C. D. (2018). ¿Influye en la felicidad la relación entre alumnos de NEE y de aula ordinaria?. International Journal of Developmental and Educational Psychology: INFAD. Revista de Psicología, 1 (3), pp. 31-40

Khoury, B. et al. (2013). Mindfulness-based therapy: a comprehensive meta-analysis. Clin Psychol Rev, 33 (6), 763-771

Las personas religiosas son más felices. (2014). Actualidad RT. Recuperado de: https://actualidad. $\underline{\text { rt.com/sociedad/161615-religion-gente-iglesia-dios-estudio }}$ 
Montenegro Castillos, M., Córdoba, P. y García Payares, A. D. (2017). Caracterización del Bienestar Subjetivo mediante la aplicación de la Escala de Satisfacción Vital de Ed Diener en estudiantes de la Corporación Universitaria Antonio José de Sucre en Sincelejo durante el primer semestre del año 2015. Encuentros, 15 (2), pp. 145-156.

Ordine, N. y Flexner, A. (2013). “L'utilità dell'inutile: manifiesto”. Giunti, Recuperado de https:// elpais.com/elpais/2016/01/22/eps/1453483676 726569.html

Ortiz Carmona, J.A. (2017). Docere, delectare et moveré: la importancia educativa de la Semana Santa en la sociedad contemporánea. En: Fernández Paradas, A.R. y Fernández Paradas, M. (Coords.) La Didáctica de las Ciencias Sociales ante la necesidad de nuevas narraciones en el siglo XXI. Digitalidad, nuevas tecnologías y competencias documentales (126-127). Granada: Comares.

Oswald, A. J., Proto, E. y Sgroi, D. (2015). Happiness and productivity. Journal of Labour Economics, 33 (4), 789-822.

Pulido Acosta, F. y Herrera Clavero, F. (2018). Predictores de la Felicidad y la Inteligencia Emocional en la Educación Secundaria. Revista Colombiana de Psicología, 27 (1), pp. 71-84.

Randstad. (2016). Randstad Workmonitor, q1 2016 report. Recuperado de: https://workforcein$\underline{\text { sights.randstad.com/hr-research-reports-workmonitor-q12016 }}$

Ravina Ripoll, R., Bayardo Tobar Pesántez, L. y Galiano Coronil, A. (2018). Labor Hapiness in public organizations. The case of the local pólice of San Fernando (Cádiz). En: Ravina Ripoll, R., Bayardo Tobar Pesántez, L. y Galiano Coronil, A. (Coords.). Claves para un desarrollo sostenible. La creatividad y el happiness management como portafolio de la innovación tecnológica, empresarial y marketing social. Granada: Comares.

Ravina Ripoll, R., Villena Manzanares, F. y Gutiérrez Montoya, G. A. (2017). Una aproximación teórica para mejorar los resultados de innovación en las empresas desde la perspectiva de "Hapiness Management". Revista de Ciencias de la Administración y Economía, 14.

Reino Unido: los creyentes son más felices que los ateos. Protestante digital. Recuperado de http:// protestantedigital.com/sociedad/38523/Reino Unido los creyentes son mas felices que los ateos

Salazar, J.E.E., Larrucha Huansi, U.R. y Flores Esteba, I.E. (2016). Felicidad: en estudio comparativo en estudiantes universitarios de Psicología y Administración de una universidad pública en Tarapolo". Apuntes Psicológicos, 1, 21-29. 
Salgado, G. (2006). Felicidad en estudiantes de universidades de Perú, Bolivia, Paraguay y Argentina. Revista Cultural de la Universidad de San Martín de Porres, 20, 531-553.

Santamaría Cárdaba, N. (2018). Educando para el desarrollo y la ciudadanía a través de la psicología positiva. ReiDoCrea: Revista electrónica de investigación y docencia creativa, 7 , pp. 98-109.

Solanos Gallegos, S. (2018). Prólogo. En: Ravina Ripoll, R., Bayardo Tobar Pesántez, L. y Galiano Coronil, A. (Coords.). Claves para un desarrollo sostenible. La creatividad y el happiness management como portafolio de la innovación tecnológica, empresarial y marketing social. Granada: Comares.

Vargas Guillén, G. y Guerrero, M. J. (2017). La puesta en vilo de las humanidades: juego y resistencia. Revista Colombiana de Educación, 72, pp. 39-63.nes los padres también se implican en la misma medida, y en el caso de familias separadas ambos padres se involucran de forma equitativa. 